\title{
Guidelines for the Management of Snakebites
}

\author{
${ }^{1}$ Gurinder Mohan, ${ }^{2}$ Arvinderpal Singh, ${ }^{3}$ Trimaan Singh
}

\begin{abstract}
India is estimated to have the highest snakebite mortality in the world. World Health Organization (WHO) places the number of bites to be 83,000 per annum with 11,000 deaths. ${ }^{1}$ Most of the fatalities are due to the victim not reaching the hospital in time where definite treatment can be administered. In addition, community is also not well informed about the occupational risks and simple measures which can prevent the bite. It continues to adopt harmful first-aid practices, such as tourniquets, cutting, and suction. Studies reveal that primary care doctors do not treat snakebite patients mainly due to lack of confidence. ${ }^{2}$ At the secondary and the tertiary care level, multiple protocols are being followed for polyvalent anti-snake venom (ASV) administration, predominantly based on western textbooks.
\end{abstract}

Keywords: Anti-snake venom, First aid, Snakebite.

How to cite this article: Mohan G, Singh A, Singh T. Guidelines for the Management of Snakebites. Curr Trends Diagn Treat 2018;2(2):102-108.

Source of support: Self

Conflict of interest: None

\section{SNAKES OF INDIA}

There are about 236 species of snakes in India, most of which are nonpoisonous. Their bites, apart from causing panic reaction and local injury, do not harm the patients. However, there are 13 known species that are poisonous, of these, four, namely common cobra (Naja naja), Russell's viper (Daboia russelii), saw-scaled viper (Echis carinatus), and common krait (Bungarus caeruleus), are highly venomous and believed to be responsible for most of the poisonous bites in India. ${ }^{3}$ However, this assumption of great four has led to non-identification of other poisonous species which are going unnoticed and leading to deaths. The recent discovery of the hump-nosed viper, capable of causing life-threatening symptoms, has demonstrated this. This nonrecognition has led the ASV manufacturers

${ }^{1}$ Professor and Head, ${ }^{2}$ Associate Professor, ${ }^{3}$ Senior Resident

${ }^{1,3}$ Department of Medicine, Sri Guru Ram Das Institute of Medical Sciences \& Research, Jalandhar, Punjab, India

${ }^{2}$ Department of Anaesthesia, Sri Guru Ram Das Institute of Medical Sciences \& Research, Jalandhar, Punjab, India

Corresponding Author: Trimaan Singh, Senior Resident, Department of Medicine, Sri Guru Ram Das Institute of Medical Sciences \& Research, Jalandhar, Punjab, India, Phone: 9855358989, e-mail: trimaan.sikand@yahoo.com to produce antivenom only against these four species. ${ }^{4}$ Thus, there is a need to abandon the old concept of "The Big Four" in order to determine all the medically significant species in India.

Snake venoms comprises $>100$ different proteins: enzymes, nonenzymatic polypeptide toxins, and nontoxic proteins. Enzymes are digestive hydrolases, hyaluronidase (spreading factor), yellow L-amino acid oxidases, phospholipases A2, and peptidases. Snake venom metalloproteases (SVMPs) damage basement membranes, causing endothelial cell damage and spontaneous systemic bleeding. Procoagulant enzymes are thrombin-like, splitting fibrinogen, or activators of factors $\mathrm{V}, \mathrm{X}$, prothrombin, and other clotting factors, causing disseminated intravascular coagulation (DIC), consumption coagulopathy, and incoagulable blood. Phospholipases A2 damage mitochondria, red blood cells, leucocytes, platelets, peripheral nerve endings, skeletal muscle, vascular endothelium, and other membranes. It produces presynaptic neurotoxic activity, cardiotoxicity, myotoxicity, tissue necrosis, hypotension, hemolysis, anticoagulation, hemorrhage, plasma leakage (edema formation), and autopharmacological release of histamine and other autacoids. Polypeptide postsynaptic $(\alpha)$ neurotoxins bind to acetylcholine receptors at the motor endplate. Presynaptic ( $\beta$ ) neurotoxins are phospholipases that damage nerve endings irreparably. The composition and antigenicity of snake venoms vary greatly between and within species, maturity, seasonally, between sexes, and throughout the geographical range. Thus, envenoming by a particular species in one part of its geographical range may not be responsive to antivenom raised against venom from the same species in another area. "Dry bites": about 50\% of venomous snakebites do not result in envenoming.

\section{CLINICAL PRESENTATION}

General: Fear and anxiety are very common and can lead to panic and trauma related to falls due to severe anxiety.

Local envenoming:

- Local pain at the site of the bite (krait bites are usually painless),

- Local swelling spreading proximally,

- Tenderness,

- Painful swelling of regional lymph nodes draining bite site, 

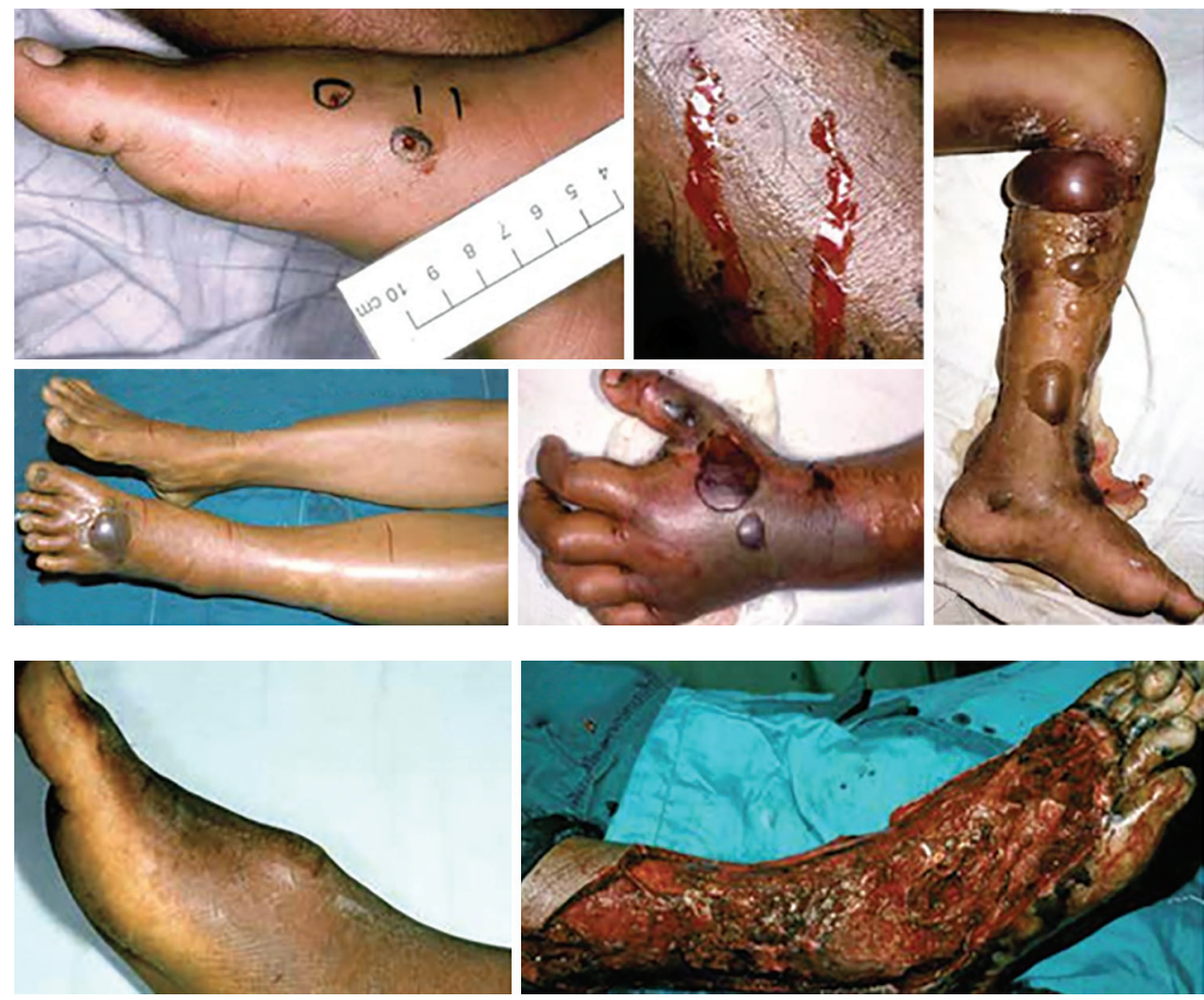

Fig. 1: Signs of local envenomation (Copyright DA Warrell)

- Other signs: fang marks, persistent local bleeding, bruising, lymphangitis, inflammation (swelling, redness, heat), blistering (blebs, bullae, vesicles), infection, abscess formation, necrosis (Fig. 1).

Systemic envenoming: nausea, vomiting, malaise, abdominal pain, weakness, drowsiness, prostration.

Cardiovascular (Viperidae):

- Hypotension, syncope, collapse, shock,

- Cardiac arrhythmias,

- Myocardial damage.

Generalized increase in capillary permeability:

- Facial, periorbital, conjunctival edema (chemosis),

- Pleural and pericardial effusions, pulmonary edema,
- Massive albuminuria,

- Hemoconcentration.

Bleeding and clotting disorders (Viperidae):

- Local traumatic bleeding from recent and partly healed wounds and venepuncture sites;

- Spontaneous systemic bleeding (gums, epistaxis, hemoptysis, meningism from subarachnoid hemorrhage, lateralizing signs, and/or coma from cerebral hemorrhage/thrombosis), hematemesis, rectal bleeding or melena, hematuria, vaginal bleeding, subconjunctival hemorrhages, skin petechiae, purpura, discoid hemorrhages, ecchymoses (Fig. 2).

Neurological (Elapidae, Viperidae, e.g., Russell's viper D. russelii, and Gloydius species) (Fig. 3):
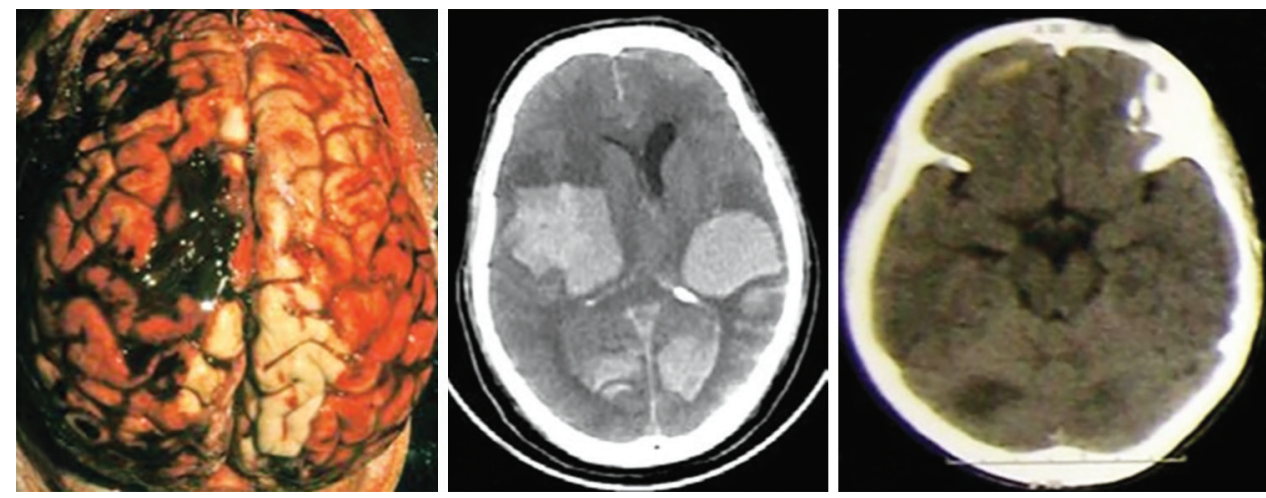

Fig 2: Intracranial bleed (Copyright Dr Suvarna Patil) 


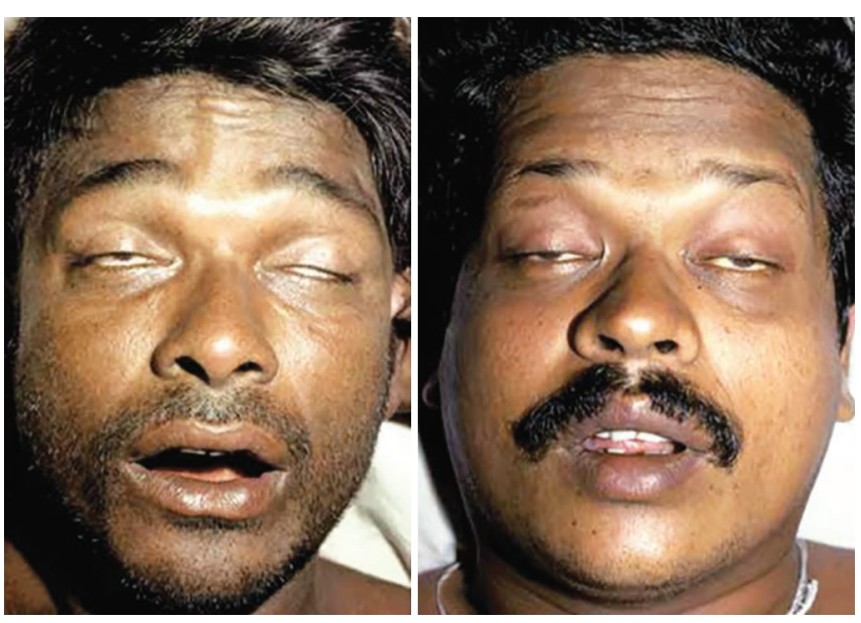

Fig. 3: Neurological manifestation-Ptosis (Copyright DA Warrell)

- Bilateral ptosis,

- External ophthalmoplegia,

- Descending paralysis progressing to generalized flaccid paralysis.

\section{Generalized rhabdomyolysis:}

- Muscular stiffness, tenderness,

- Painful on passive stretching, trismus,

- Dark brown urine (Fig. 4).

Acute kidney injury:

- Loin (lower back) pain,

- Hematuria, hemoglobinuria, myoglobinuria,

- Oliguria/anuria,

- Uremia (acidotic breathing, hiccups, nausea, pleuritic chest pain, encephalopathy).

Pituitary insufficiency (Russell's viper) (Fig. 2):

- Acute-shock, hypoglycemia;

- Chronic-weakness, loss of secondary sexual characters, loss of libido, amenorrhea, testicular atrophy, hypothyroidism, etc.

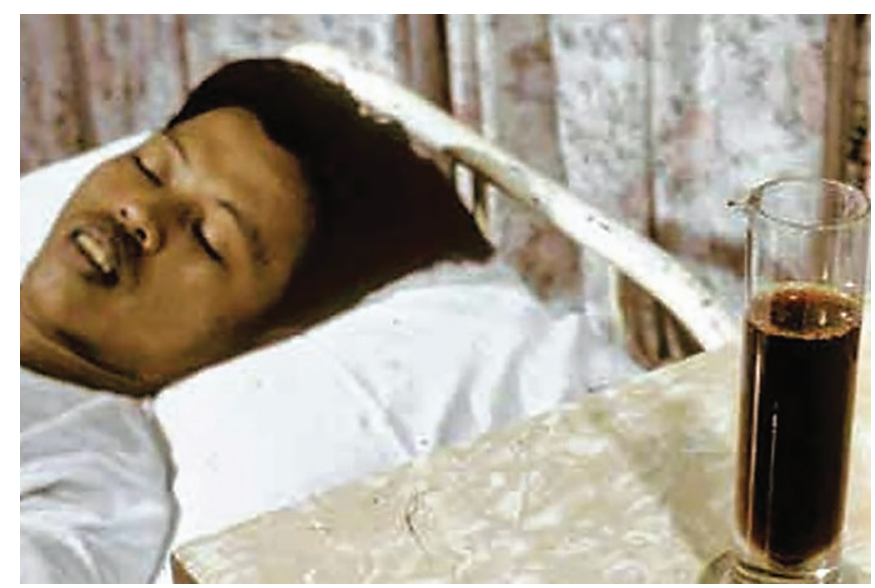

Fig. 4: Myoglobinuria and hemoglobinuria (Reid, 1979; Warrell, 1994)

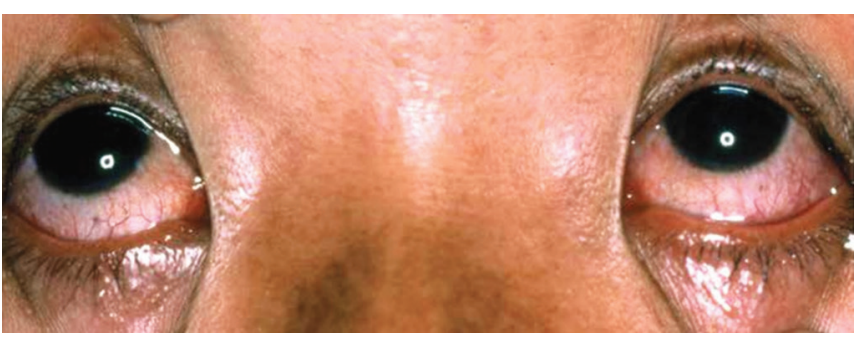

Fig. 5: Conjunctivitis associated with spat of venom (Copyright DA Warrell)

If the "spat" venom enters the eyes, there is immediate and persistent intense burning, stinging pain, followed by profuse watering of the eyes with the production of whitish discharge, congested conjunctivae, spasm and swelling of the eyelids, photophobia, clouding of vision or temporary blindness, corneal ulceration, permanent corneal scarring, and secondary endophthalmitis (Fig. 5).

\section{INVESTIGATIONS/LABORATORY TESTS}

20-minute whole blood clotting test (20WBCT) is a simple, informative bedside test requiring only a new, clean, dry, ordinary glass tube, bottle, vial, or syringe. The positive (nonclotting) result indicates severe consumption coagulopathy and the need for immediate antivenom treatment. False positive (nonclotting) 20WBCT results from the use of plastic, polystyrene, or polypropylene rather than ordinary glass, or glass cleaned with detergent, soap, or washing fluid that destroy surface activation of blood coagulation. The test should be carried out every 30 minutes from admission for 3 hours and then hourly after that. If incoagulable blood is discovered, the 6 hourly cycle is then adopted to test for the requirement for repeat doses of ASV (Fig. 6).

Other more sensitive laboratory tests of blood coagulation:

- International normalized ratio (INR) based on prothrombin time (PT) (> or $=1.2$ is abnormal).

- Activated partial thromboplastin time (aPPT), fibrin(ogen)-related antigens (fibrin degradation products-FDP), or D-dimer.

- Hemoglobin concentration/hematocrit, fragmented red cells ("helmet cell", schistocytes) signifying microangiopathic hemolysis.

- Thrombocytopenia.

- Neutrophil leukocytosis.

- Observe spontaneously sedimented plasma for hemoglobinemia/myoglobinemia.

- Biochemical abnormalities in plasma creatinine, urea/ blood urea nitrogen, and potassium concentrations occur in acute kidney injury (Russell's viper, humpnosed pit-viper, and sea-snake envenoming).

- Elevated aminotransferases and muscle enzymes (creatine kinase, aldolase, etc.) indicate local and 


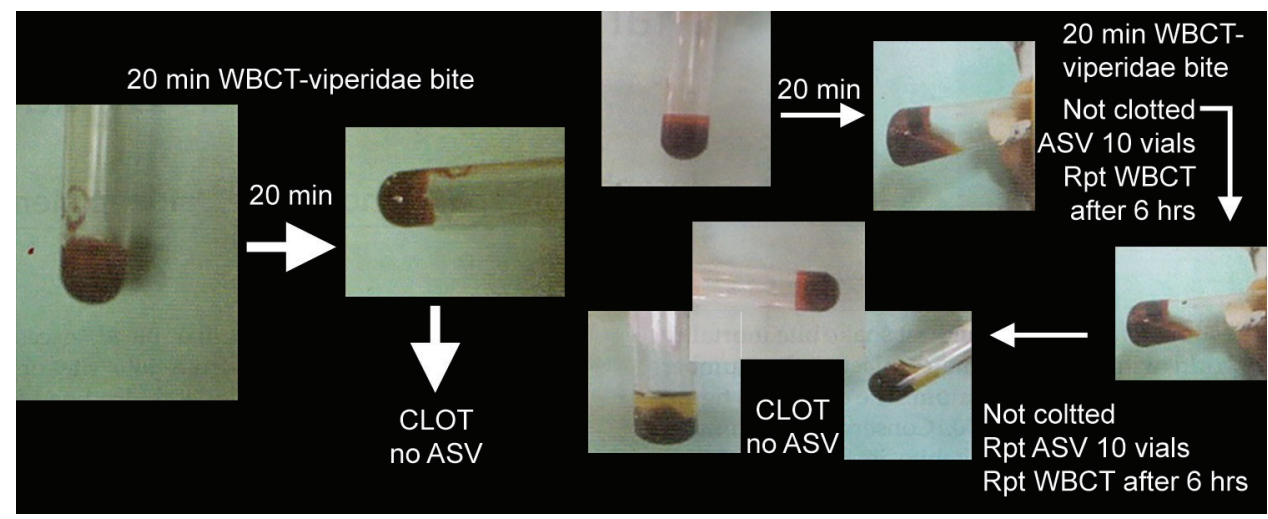

Fig. 6: 20-minute whole blood clotting test (20WBCT)

generalized muscle damage (sea snakes, some kraits, some Australasian Elapidae, and Russell's viper bites).

- Hyponatremia is associated with krait bites.

- Urine examination: dipsticks test for blood hemoglobin, myoglobin, and proteinuria.

- Urine microscopy to detect erythrocytes and red cell casts, indicating glomerular bleeding and eosinophilia suggesting acute interstitial nephritis.

Other investigations:

- Chest radiography for detecting pulmonary edema, hemorrhages, infarcts, pleural effusions, and secondary bronchopneumonia.

- Ultrasound for assessing local envenoming, deep vein thrombosis, pleural and pericardial effusion, and bleeding.

- Echocardiography for myocardial dysfunction.

- Computed tomography (CT) and magnetic resonance imaging (MRI) for intracranial and spinal hemorrhages and infarcts and osteomyelitis at the bite site.

- ECG for arrhythmias, myocardial damage, and evidence of hyperkalemia.

Also, the single breath counting test is done in suspected bites with signs of neurological involvement and the same is repeated at 15 minute interval over the first 2 hours.

\section{MANAGEMENT PROTOCOL (AS PER RECENT WHO GUIDELINES 2016)}

First-Aid Treatment ${ }^{8}$

The first aid being currently recommended is based on the mnemonic.

"CARRY NO R.I.G.H.T."

It consists of the following:

CARRY = Do not allow victim to walk even for a short distance; just carry him in any form, especially when bite is at leg.

No $=$ Tourniquet $^{5} ;$ No-electrotherapy $^{7}$; No-cutting; No-pressure immobilization; No-sucking of venom ${ }^{6}$
$\mathbf{R}=$ Reassure the patient. About $70 \%$ of all snakebites are from nonvenomous species. Only $50 \%$ of bites by venomous species actually envenomate the patient.

I = Immobilize in the same way as a fractured limb. Use bandages or cloth to hold the splints, not to block the blood supply or apply pressure. Do not apply any compression in the form of tight ligatures, they do not work and can be dangerous!

GH = Get to hospital immediately. Traditional remedies have NO PROVEN benefit in treating snakebite.

$\mathbf{T}=$ Tell the doctor of any systemic symptoms that manifest on the way to hospital.

Traditional methods should be discarded.

\section{Pain}

Snakebite can often cause severe pain at the bite site. This can normally be treated with painkillers, such as paracetamol. Aspirin and nonsteroidal anti-inflammatory drugs (NSAIDs) should not be used, as they can exacerbate bleeding. This can be particularly dangerous in a patient already having coagulopathy. Mild opiates, such as tramadol $50 \mathrm{mg}$, can be used for good pain relief.

\section{Handling Tourniquets}

Care must be taken when removing tight tourniquets tied by the victim. Sudden removal can lead to a massive surge of venom leading to neurological paralysis and hypotension due to vasodilation. Before removal of the tourniquet, test for the presence of a pulse distal to the tourniquet. Be prepared to handle complications, such as sudden respiratory distress or hypotension. If the tourniquet has occluded the distal pulse, then a blood pressure cuff can be applied to reduce the pressure slowly. ${ }^{9}$

\section{ASV}

ASV is the mainstay of treatment. In India, polyvalent ASV, i.e., effective against all the four common species: Russell's viper, common cobra, common krait, and sawscaled viper, and no monovalent ASVs are available. There are known species, such as the hump-nosed viper 
(Hypnale hypnale), where polyvalent ASV is ineffective. ASV is produced both in liquid and lyophilized forms. There is no evidence to suggest which form is more effective. Liquid ASV requires a reliable cold chain and has a 2-year shelf life. Lyophilized ASV, in powder form, has a 5 -year shelf life and requires only to be kept cool.

\section{Indications for Antivenom}

Antivenom treatment is recommended if and when a patient with proven or suspected snakebite develops one or more of the following signs:

Systemic envenoming

- Hemostatic abnormalities: spontaneous systemic bleeding distant from the bite site, coagulopathy [+ve (nonclotting) 20WBCT or other laboratory tests, such as INR $>1.2$ or patient's prothrombin time $>4$ to 5 seconds longer than laboratory control value], or thrombocytopenia [ $<1.0$ lakh per microliter of blood)].

- Neurotoxic signs: ptosis, external ophthalmoplegia, paralysis, etc.

- Cardiovascular abnormalities: hypotension, shock, cardiac arrhythmia, and abnormal ECG.

- Acute kidney injury (renal failure): oliguria/anuria and rising serum creatinine/urea.

- Hemoglobin-/myoglobinuria: dark brown urine or other evidence of intravascular hemolysis or generalized rhabdomyolysis (generalized muscle aches, pains, tenderness, and pain on passive stretching.

- Supportinglaboratory evidence of systemicenvenoming and local envenoming.

Local envenoming

- Local swelling involving more than half of the bitten limb (in the absence of a tourniquet) within 48 hours of the bite. Swelling after bites on the digits (toes and, especially, fingers).

- Rapid extension of swelling (e.g., beyond the wrist or ankle within a few hours of bites on the hands or feet).

- Development of an enlarged tender lymph node draining the bitten limb (Table 1).

\section{ASV Reactions}

The prophylactic regime is 0.25 to $0.3 \mathrm{mg}$ adrenaline 1:1000 given subcutaneously (deep IM) if the victim has a known sensitivity to ASV. Premedication with adrenaline, hydrocortisone, and antihistamine may be advisable, in order to prevent severe reactions. In the case of reactions, antihistaminics can be administered to control the reaction and if severe, adrenaline should be administered. ${ }^{10}$ Once the patient has recovered, the ASV can be restarted slowly after 10 to 15 minutes, keeping close observation. Late serum sickness can be treated with oral prednisolone and/or antihistaminics.

\section{Test Dose of ASV}

Test dose has been shown to have no predictive value in detecting anaphylactoid or late serum reactions and should not be used. These reactions are not IgE mediated but complement activated. They may also pre-sensitize the patient and thereby create greater risk.

\section{ASV Dosage ${ }^{11}$}

Symptoms and signs being not a useful guide for deciding the degree of envenomation and having no diagnostic methods to determine the level of venom in blood or tissue, any ASV regimen adopted could only be an estimate. One milliliter of ASV neutralizes $0.6 \mathrm{mg}$ of Russell's viper venom, $0.6 \mathrm{mg}$ of cobra venom, $0.45 \mathrm{mg}$ of krait venom, and $0.45 \mathrm{mg}$ of saw-scaled viper venom.

Start IV normal saline with a wide bore needle. Begin with 10 vials of ASV in $100 \mathrm{~mL}$ of normal saline and to start with 10-15 drops per minute for 15 minutes and watch for reactions. If the patient is not having signs and symptoms of anaphylactic shock, continue the ASV. All ASVs are to be administered over a 1 hour period at constant speed. Continue to monitor the vital signs at 5 minute interval for the first 30 minutes and then at 15 minute interval for 2 hours. ASV can be repeated if the following criteria are present (Table 2).

\section{MANAGEMENT OF NEUROTOXIC ENVENOMATION}

Antivenom treatment alone cannot be relied upon to save the life of a patient with bulbar and respiratory

Table 2: Criteria for repetition of ASV

Criteria for giving more antivenom

- Persistence or recurrence of blood incoagulability after 6 hours or bleeding after 1 to 2 hours

- Deteriorating neurotoxic or cardiovascular signs after 1 hour

Table 1: Clinical presentation

\begin{tabular}{llllll}
\hline Feature & Cobra & Kraits & Russell's viper & Saw-scaled viper & Hump-nose viper \\
\hline Local pain/tissue damage & Yes & No & Yes & Yes & Yes \\
Ptosis, neurological sign & Yes & Yes & No & No & No \\
Hemostatic abnormality & No & May occour & Yes & No & Yes \\
Renal complication & No & No & Yes & No & Yes \\
Response to neostigmine & Yes & \pm & No & Yes & No \\
Response to ASV & Yes & Yes & Yes & & No \\
\hline
\end{tabular}


paralysis. Death may result from aspiration, airway obstruction, or respiratory failure. A clear airway must be maintained. Once there is loss of gag reflex and pooling of secretions in the pharynx, failure of the cough reflex, or respiratory distress, a cuffed endotracheal, laryngeal mask airway, or supraglottic airway should be inserted. If this is impossible for any reason, or the need for prolonged ventilation is anticipated, a tracheostomy should be performed and cuffed tracheostomy tube should be inserted.

Neostigmine is an anticholinesterase that prolongs the life of acetylcholine and can, therefore, reverse respiratory failure and neurotoxic symptoms. It is particularly effective for postsynaptic neurotoxins, such as those of the cobra. There is some doubt over its usefulness against presynaptic neurotoxin, such as those of the krait and Russell's viper.

\section{NEOSTIGMINE TEST ${ }^{12}$}

This test involves the administration of 1.5 to $2 \mathrm{mg}$ of neostigmine IM together with $0.6 \mathrm{mg}$ of atropine IV. The patient should be closely observed for 1 hour to decide whether neostigmine is effective. The following measures are useful objective methods to asses this:

- Single breath count

- $\mathrm{mm}$ of iris uncovered (amount covered by the descending eye lid)

- Inter incisor distance (distance between upper and lower incisors)

- Length of time upwards gaze can be maintained.

- $\mathrm{FEV}_{1}$ (forced expiratory volume (in 1 second)) or forced vital capacity (FVC), if available

If the victim responds to the neostigmine test, then continue with $0.5 \mathrm{mg}$ of neostigmine IM half hourly plus $0.6 \mathrm{mg}$ atropine IV for five doses and then 2 to 12 hourly according to recovery. If there is no improvement in symptoms after 1 hour, neostigmine should be stopped.

Patients who respond convincingly can be maintained on neostigmine methylsulfate, 0.5 to $2.5 \mathrm{mg}$ every half hour up to $10 \mathrm{mg} / 24$ hours maximum for adults or 0.01 to $0.04 \mathrm{mg} / \mathrm{kg}$ every 2 to 4 hours for children by intramuscular, intravenous, or subcutaneous injection together with atropine to block muscarinic side effects. Patients able to swallow tablets may be maintained on atropine $0.6 \mathrm{mg}$ twice each day, neostigmine $15 \mathrm{mg}$ four times each day, or pyridostigmine $60 \mathrm{mg}$ four times each day.

\section{Ice Test (Fig. 7)}

The "ice pack test"13 as a "possible alternative to the Tensilon test" is recovery of ptosis in the one eye after applying an ice pad that inhibits intrinsic cholinesterase in a patient with postsynaptic blockade of neuromuscular
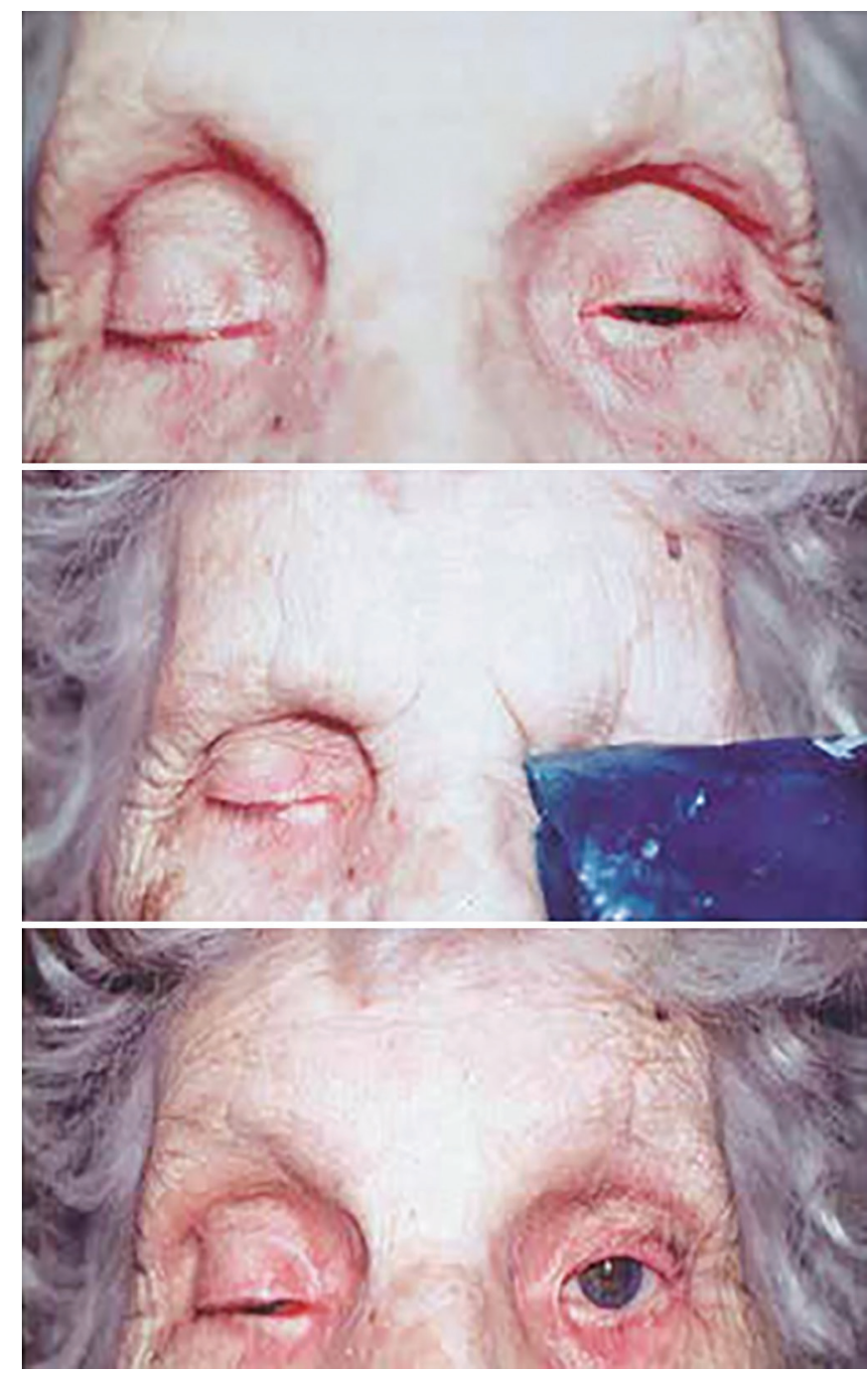

Fig. 7: Ice test for checking neurotoxicity (Delaney Y, Khooshabeh R, Benjamin L, 2002)

transmission. In this, digital pressure is applied to the frontalis muscle to avoid its influencing upper eyelid retraction. An ice-filled plastic glove or frozen ice pack is then gently applied to one eyelid for 2 minutes, after which the palpebral fissures of both eyes are immediately remeasured. A more-than-2 $\mathrm{mm}$ difference in palpebral fissure between the cooled and control eyelids might be considered a positive result.

\section{RENAL FAILURE IN SNAKEBITE}

The acute renal failure which occurs due to snakebite is multifactorial:

- Severe and persistent hypotension leading to acute tubular necrosis.

- Rhabdomyolysis and myoglobinuria.

- DIC.

- Vasculitis.

- Acute diffuse interstitial nephritis.

- Extracapillary proliferative glomerulonephritis. 
Most of the patients of acute tubular necrosis recover within few weeks, with the help of occasional need of hemodialysis, but patients who develop cortical necrosis require renal replacement therapy on a long-term basis. It is hyperkalemia rather than elevated urea and creatinine that requires dialysis. Acute kidney injury (AKI) is a hypermetabolic hyperkalemia, which may kill the patient within a few minutes and calcium gluconate and glucose-insulin are mostly ineffective. Early urgent adequate treatment with ASV can reverse the whole process of deterioration of renal function, which is far from our expectation in our country. Renal failure is a common complication of Russell's viper and hump-nosed viper bites. ${ }^{14}$ The contributory factors are intravascular hemolysis, DIC, direct nephrotoxicity, hypotension and rhabdomyolysis. Renal damage can develop very early in the case of Russell's viper bite and even when the patient arrives at hospital soon after the bite, the damage may have already been done. Studies have shown that even when ASV is administered within 1 to 2 hours after the bite, it is incapable of preventing acute renal failure (ARF).

\section{SNAKEBITE IN PREGNANCY}

Pregnant women are treated in exactly the same way as other victims. The same dosage of ASV is given. ${ }^{15}$ The victim should be referred to a gynecologist for the assessment of any impact on the fetus.

\section{SNAKEBITE IN CHILDREN}

Children receive the same ASV dosage as adults. The ASV is targeted at neutralizing the venom. Snake injects the same amount of venom into adults and children.

\section{FOLLOW-UP}

After discharge from hospital, the victim should be followed. If discharged within 24 hours, the patient should be advised to return if there is any worsening of symptoms, such as bleeding, pain, or swelling at the site of bite, difficulty in breathing, and altered sensorium. The patients should also be explained about serum sickness which may manifest after 5 to 10 days.

\section{SNAKEBITE PREVENTION}

\section{Preventive Measures}

- Walk at night with closed-type footwear (e.g., shoes or boots), and a flashlight that is switched on!

- Carry a stick when grass cutting or picking fruit or vegetables, or clearing the base of trees. Use the stick to move the grass or leaves first.

- If you encounter a snake, give the snake a chance to move away!
- Pay close attention to the leaves and sticks on the ground when collecting woods.

- Keep animal feed and rubbish away from your house. They attract rats, and snakes will eventually follow.

- Try to avoid sleeping on the ground. Keep plants away from your doors and windows. Snakes like cover, and plants help them climb up.

\section{REFERENCES}

1. Kasturiratne A, Wickramsinghe AR, DeSilva N, et al. The global burden of snakebite: a literature analysis and modelling based on regional estimates of envenoming and deaths. PLOS Med 2008;5:e218.

2. Simpson ID. A study of current knowledge base in treating snake bite among doctors in high risk countries of India and Pakistan: does snake bite treatment training reflect local requirements? Trans R Soc Trop Med Hyg 2008;102:1108-1114

3. Warrell DA. WHO/SEARO guidelines for the clinical management of snakebite in the southeast Asian region. SE Asian J Trop Med Pub Health 1999;30:1-85.

4. Simpson ID, Norris RL. Snakes of medical importance: is the concept of big four still relevant and useful? Wilderness Environ Med 2007;18:2-9.

5. Amaral CF, Campolina D, Dias MB, et al. Tourniquet ineffective-ness to reduce the severity of envenoming after Crotalus durissus snake bite in Belo Horizonte, Minas Gerais, Brazil. Toxicon 1998;36:805-808.

6. Bush SP, Hegewald KG, Green SM, et al. Effects of a negativepressure venom extraction device (Extractor) on local tissue injury after artificial rattlesnake envenomation in a porcine model. Wilderness Environ Med 2000;11:180-188.

7. Davis D, Branch K, Egen NB, et al. The effect of an electrical current on snake venom toxicity. J Wilderness Med 1992;3: 48-53.

8. Anker RL, Staffon WG, Loiselle DS, et al. Retarding the uptake of "mock venom" in humans. Comparison of three first-aid treatments. Med J Australia 1982;1:212-214.

9. Simpson ID. Snakebite Management in India, the first few hours: a guide for primary care physicians. J Indian Med Assoc 2007;105:32435.

10. McLean-Tooke AP, Bethune CA, Fay AC, et al. Adrenaline in the treatment of anaphylaxis: what is the evidence? BMJ 2003; 327:1332-1335.

11. Srimannanarayana J, Dutta TK, Sahai A, et al. Rational use of antisnake venom (ASV): Trial of various regimens in hemotoxic snake envenomation. J Assoc Phys India 2004:52:788-793.

12. Anil A, Singh S, Bhalla A, et al. Role of neostigmine and polyvalent antivenin in Indian common krait (Bungarus caeruleus) bite. J Infection Public Health 2010;3:83-87.

13. Kearsey C, Fernando P, D'Costa D et al. The use of the ice pack test in myasthenia gravis. JRSM Short Reports 2010;1(1):14. doi: 10.1258/shorts.2009.090037.

14. Chugh KS. Snake-bite-induced acute renal failure in India. Kidney Int. 1989 Mar;35(3):891-907.

15. Brown SA, Seifert SA, Rayburn WF. Management of envenomations during pregnancy. Clin Toxicol (Phila) 2013 Jan;51(1):3-15. doi: 10.3109/15563650.2012.760127. 